\title{
Mobile Augmented Integrated Framework for Citizen Centric E-Governance Services- MAIFCCES
}

\author{
Shailesh Khanesha \\ National Informatics Centre(NIC), Govt. of India, Gandhinagar (Gujarat), 382010, India, \\ E-mail: shailesh.khanesha@nic.in \\ Dr. Ashish Jani \\ Faculty of Comp. Sci., KSV University, Gandhinagar (Gujarat), 382023 India, \\ E-mail: aashishjani@yahoo.com
}

\begin{abstract}
The increasing adoption of improving mobile technologies has opened up a window for research and innovative services development opportunities in diversified dimensions. Government system anticipates delivery of citizen centric services through hand held devices in an integrated manner. This paper has made an attempt to develop user friendly citizen centric interface to avail government services in faster and cost-effective way through mobile device. The proposed MAIFCCES framework provides an interface for citizen to apply for government services and monitor it's status through mobile device. Also suggest a mechanism to process and monitor the applications of citizen centrally in government data centre.
\end{abstract}

Index Terms-MGovernance, eGovernance, mService, Citizen, Mobile Apps, RESTFul.

\section{INTRODUCTION}

The increasing adoption of improving smart phone mobile technologies has open up a window for research and innovative service delivery mechanism from government to citizen. With advent of digital era and mobile technology, also it has got the support of ICT, strategies has changed greatly which has not only opened up the dimension of utility but improved efficiency of service delivery at various stages and has put a trust to government service delivery for citizen in an integrated environment rather than distributed environment. Also penetration rate of mobile handsets among users in the country is ever increasing and it has added the dimension of "information axis and its users Anywhere Anytime".

Thought e-Government is on digital framework but still is constraint during mobility. To provide the facility of governance event to the players of governance during their mobility, m-Governance framework is needed. The desired framework of $\mathrm{m}$-Governance is intended to provide additionally the services of land records, health, public grievances, certificates, etc. There are two important issues related to the transmission from e-
Government to m- Government: 1) M-Government is inevitable because of the citizen's expectations for a better and convenient government services. 2) MGovernment is complementary to e-government because some of the e-government services can be replaced by mgovernment services on mobile platform. Basic mGovernment applications should be keystones of wireless strategies for governments worldwide [1].

Government understands that the use of mobile devices and tools is an immediate challenge. Based on the interview from the various agency along with the assessment framework and gap analysis, several key recommendations are suggested for mobile governance in [2] are 1) Define requirement for several use cases 2) Establish a cross-functional team to evaluate the technical, legal, privacy and other factors associated with the use of government resources 3) Continue development of a mobile computing decision framework which adds a methodical approach to determining mobile solution implementation 4) Develop a policy and guidance to support more flexible use of commercial mobile devices, and develop an acquisition strategy for procurement of mobile technologies that comply with Government-wide policy, emerging standards and requirements and 5) Continue development of requirements and recommendations to enable the use of strong authentication and encryption to accelerate the use of mobile devices in a secure manner.

Governments are moving to improve their services by adding mobile government (m-government) as a new delivery channel by utilizing the available wireless infrastructure installed by private mobile operators. Mobile communications and Internet technologies are enabling access to new e-government services at any time and from anywhere. M-government is considered as a subset of e-government comprising another channel to provide governmental information and services. General goals mentioned in [3] of m-Government can be considered as 1) increasing added values of egovernment services and 2) implementing public services by creating convergence between internet services and wireless services. From citizen points of view, m- 
Government provides full-scale access to services of public sector and relevant sectors. However, mgovernment is still in its early stages and very few governments have implemented full mobile government services. Planning and implementation of projects related to mobile government based on the current research in the public administration is the best way to improve public services; More attention and concentration is also necessary on effectiveness of governance and public services delivery, accountability and responsiveness as well as informing indicator [3].

Since last few years a set of m-governance framework for public service delivery has been developed and used. It has also been observed that the mobile governance of later period has simplified the government service delivery mechanism. However further improvement in $\mathrm{m}$-Governance system from the existing stage of mobile governance enable to work rigorously to enhance the functionality as well as improving the parameters like Security, Authenticity of Data, Performance, CostEffectiveness, Scalability, Integration of number of services, language selection and user friendly interface for wider acceptance. The services incorporated in this framework are in an integrated manner which involves almost all the government department's services to the citizen. The framework design provides the scalability to add more number of services as well. Performance has been improved through use of native mobile application and data communication by RESTful web service architecture.

This research aims to identify, describe and produce an integrated mobile augmented framework that can promote the use of mobile phones for increased citizen participation and successful delivery of government services. There are below objectives are set for undertaking this research.

- To evaluate how mobile phone technology can be used as a tool for government service delivery.

- Addressing the issues of performance, scalability, integration of services, language dominance and cost-effectiveness.

- Better public service delivery mechanism from government to citizen(G2C)

- Integration of government services for citizen on single mobile interface and central monitoring by government authority.

The rest of the dissertation is organized in the chapters of literature review, proposed framework, methodology, result and conclusion with future extension of work. Literature review chapter gives a brief survey of relates works. Chapter of proposed framework depicts the proposed model for mobile governance. Chapter of methodology describe the prototype development, implementation and validation. Result chapter shows the implementation result of prototype model and the last chapter specifies the conclusions and indicates the recommendation for future research.

\section{RELATED WORK}

Sabrish.K et.al [4] has undertaken the issues of public service delivery under e-Governance to achieve a revolutionary change in the form of mobile government services by using cloud based interoperable framework. They have proposed scalable cloud enabled mGovernance framework on the principles of Service Oriented Architecture(SOA) and using standards defined by W3C, XML, WSI and SOAP to incorporate new services on the fly as in software as a service platform. Implementing the proposed work they have achieved sharing of common ICT infrastructure, Integration of mobile applications and mobile network services (Voice, SMS, MMS, USSD, GPRS, 3G and LBS), also consistent and standardize interface. The challenge ahead of their work is the security mechanism to handle the evolving threads.

Aarathi Prasad et.al [5] has undertaken the issues related to trust and accuracy of health information collected from sensors human entered data to achieve accurate and authenticate information received on mobile phones. They have proposed provenance framework for mHealth devices by collecting and sharing of provenance metadata (information about origin and changes made to it) based on pre-defined rules which remain stored in rules files on the user's mobile phone. Implementing the proposed work data consumer can verify the certain provenance property of information they received on mobile phones to make sure that the information is accurate and trusted. The challenges ahead of their work is to provide modeling framework for developer and domain experts to construct and validate this model, which also include graphical interface for recipient to view and verify the provenance property of data received.

Richard K. Lomotey et.al [6] has undertaken the issues to access medical data on mobile devices in real-time mode in case of mission critical and decision making situations. They focus the challenges related to mobile environment such as wireless connectivity, fluctuating bandwidth, various types of devices, device battery life limitations and threats to privacy of data by proposing a middleware oriented mobile cloud computing framework to achieve real-time data propagation and privacy and security options while accessing medical data in mobile devices. Provenance concept, data encryption and REST standards are employed in the proposed designed for security and performance achievement. Implementing the proposed framework they have found communication overhead due to data protection and security features such as encryption, decryption, marshaling and demarshaling of data, but user experience not affected that much because of about 1 second is worse recorded time. The challenges and future work ahead of their work is to improve communication performance and the automation of location access as well as to provide scalability of the middleware hosted on private cloud.

Desta Mengistu et.al [7] has discuss the challenges ahead in mobile governance such as complexity of various mobile technologies, secure delivery of reliable services, peoples readiness, legal issues, 
compatibility(Standardize contents provides to citizen), battery life limitation of mobile devices. Also discuss the opportunities of $\mathrm{m}$-government and recommended to prepare a priority list of m-services for rapid implementation by government.

Shashank Garg et.al [8] has undertaken the issues of early detection of various disease (Malaria, Typhoid, TB, Leprosy, Tetanus, Measles, etc.) and medical intervention system for integrated disease surveillance in India in the domain of public health. They have proposed mobile computing framework by mapping the manual process onto mobile device operated by primary health-care workers, health officers and district/state health monitoring team. Implementing the proposed framework they have achieved early access of surveillance data, replace card based manual reporting and user friendly data capturing screen which significantly improve the productivity of primary health-care workers. The future of scope of development is suggested to implement in other developing countries which may require little customization in the framework developed.

Diatha Krishna Sunder et.al [9] has undertaken the issues of public service delivery mechanism in Indian urban local bodies to achieve faster, cost effective and scalable solutions for delivery of services at the door steps of citizen by using mobile technologies. They have proposed a framework for mobile governance in urban local bodies in the Indian context. In the framework, they have used semantic web for software service portability through web services, Information management and sharing through ontology, Mobile device for access of services in hand, information security and user authentication. Implementing the proposed framework they have achieved the faster, cost effective and quality service delivery such as Property registration, urban transport, Solid waste management, Utility bills like Electricity, water, gas, etc. The future scope of development is suggested to prepare scalable and more cost-effective framework which can be replicable in other countries.

Emmanuel Eilu et.al [10] has undertaken the issues of low voting percentage during elections in developing countries. They have highlighted the use mobile phone and ICT (Information and Communication Technology) for voting purpose and study the case of M-voting project of Uganda by using quantitative approach where 900 questionnaire given to respondents of different age group (from 18 and above)., In the study they have found that $60 \%$ of those who owned mobile phones were not willing to use mobile phone for voting due to political, technological, social and cultural reasons. The authors mentioned the aspects of future work and challenges to achieve wider acceptance of mobile phones for voting are elimination of election fraud, mobile phone network performance, existing laws and policies on elections and using mobile phones as voting facilitators.

Government of India [11] address the issues of delivery of public information and services to all citizens and business in the country to achieve wider reach and access to public services to all citizens in the country especially in the rural areas. Department of Electronics and Information Technology (DeitY), Government of India conceptualized and formulated mobile governance platform for all government department and agencies of country for delivery of public services to citizens and business on mobile devices. Government of India [12] designed and declared framework for mobile governance to utilize the massive reach of mobile phones and exploit the potential of mobile applications and technology to enable easy and round the clock access of public services, especially in the rural areas. The aim of this framework is to create unique infrastructure as well as application development ecosystem for m-Governance in the country. Measures in this framework are

- All government web sites shall be made mobilecompliant and using One-Web approach.

- Use of open standards to developed mobile applications for interoperability of applications across various operating systems and devices

- Uniform pre-defined mobile numbers(long and short codes) shall be used for convenience

- All government departments and agencies shall provide public services on mobile devices. Also define service levels for such services.

Government of Karnataka launched Karnataka Mobile One platform for better and efficient public service delivery through mobile devices. This platform integrates all services provided by government in single application that can run on mobile device also. It is a first such initiative in the country India. It is an unified mobile platform services which is accessible on Android smart phones or iOS smart phones and basic handsets through SMS, IVR (Integrated voice response) and mobile web (Responsive web application) [13,14]. Government of Utter Pradesh launched an integrated mobile platform developed by CDAC Mumbai named UPOne is mobile application. This application facilitate the citizens for enquiring status of various applications submitted, Checking the authenticity of certificates issues to the citizen and display dashboard of various application summary on mobile platform [15].

From the analysis of related work it is clear that integration and inter linking of number of government services delivery on mobile platform are required with good performance, security and on cost effective way. But as mentioned in [6] that by adding an encryption technology while communicating for security purpose, it degrades the performance of mobile application, thus one parameter is affecting the performance of other parameters. Fig. 1 shows the parameters which exists scope of improvement in the existing frameworks.

Therefore, a proposed $\mathrm{m}$-Governance framework is suggested to achieve an integration of various mGovernance services on single mobile platform with 1) Communication performance and security by using RESTful web services, 2) Authenticity of Data using authorization and authentication, 3) Enhancement in performance and Cost-effectiveness by using native 
features of mobile phones and open standards in development process, 4) Identification of number of services to be deliver on mobile devices and 5) Selection of operating language for better understanding of citizen and wider acceptance.

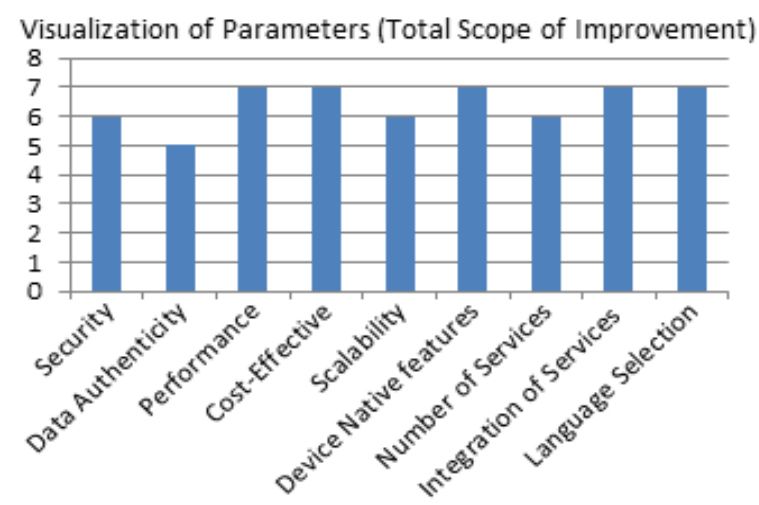

Fig.1. Scop of Improvement

\section{MAIFCCES - PROPOSED FRAMEWORK}

The literature review findings and subsequent analytics to ascertain parameters to be considered with their existing levels need and enhancement. A framework is needed to be proposed to facilitate mobile governance. The functionalities are to be embedded in the existing domain of functionalities with a suitable integration and citizen centric interface to render services in smarter way with lesser cost. The technological growth of mobile computing have widen the window rendering services through Mobile Apps, do empower citizen but lacks in integration and some functionalities along with lesser ease of user interface from uses point of view.

The proposed architecture MAIFCCES (Mobile Augmented Integrated Framework for Citizen Centric eGovernance Services) is an integrated architecture for mobile governance on the re-use of every government departments to incorporate its citizen centric service delivery through mobile device.

The citizen centric module suggested in this framework contains mobile application through which citizen can prepare application to avail government services and send it online to the government authority when gets connectivity. This module provides an integration of number of government services provided by the government to the citizen.

Another module in this framework is in backend and act as a server side module which contains Web Service repository based on REST architecture to receive data of application from mobile client and store into central database. Also provides application status to mobile client as and when access. The status of application is updated by corresponding e-Governance application which is already running in the government departments such as Land Record Computerization, Public Grievances Redressal Application, Jan Seva Kendra, etc. Therefore, this module is middle level service provider which communicates with both client side $\mathrm{m}$-Governance application and e-Governance application running on government departments.
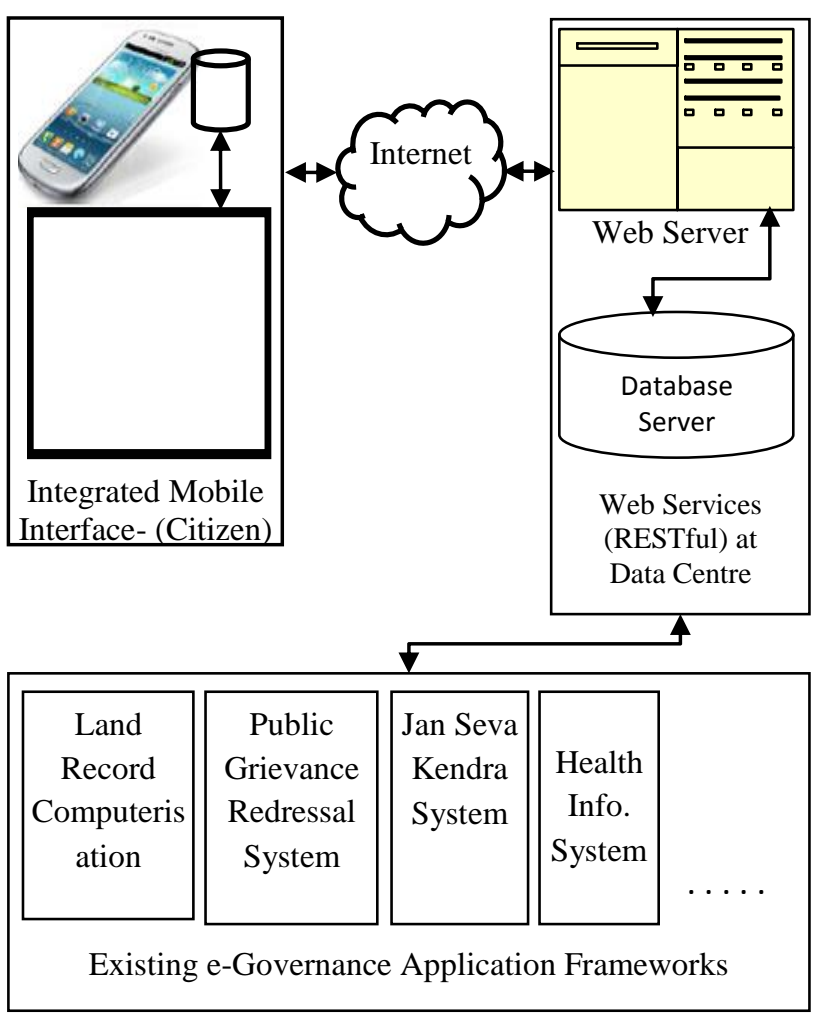

Fig.2. Architecture of Framework

As indicated in fig. 3, data from mobile device moves to registration database through RESTful web service repository and other existing e-governance system have to access this data in order to process the citizen application.

\section{MethodolOGY}

The proposed framework is implemented through a prototype of the implementation architecture that demonstrates the use of a subset of the functions identified in the mobile governance framework. The prototype is a mobile governance system: Citizen centric module to apply for government services and to avail information of related government records (e. g. Land Record, Health Card, etc.) for verification as well as get the status or progress of application. The submitted applications on remote server are processed via a simple workflow that provides approval or rejection for application after verification of document received. The implementation provides user interfaces for online application through mobile device and access of records from government authorities and web service API to communicate with mobile device and remote servers.

\section{A. Technology Adoption}

Langrial, Sitwat et.al [16] has indicated the technology of native mobile applications to develop efficacious 
persuasive mobile application. It can help designers to build behavior change support systems rather than standalone persuasive applications. Andre Charland et.al [17] has indicated that native mobile application and mobile web are pitted against one another and likely outcome is hybrid solution to balance the web vs. native apps based on an application's primary objectives, development and business requirements. If web does not fulfill a capability of particular application requires, native with web service consuming option is an exciting opportunity to contribute and close the Web/Native chasm in the process. Paul Boag [18] has explained the four options to deal with mobile device in different circumstances. These options are Responsive Website, Native Application, Web Application and Hybrid Application.

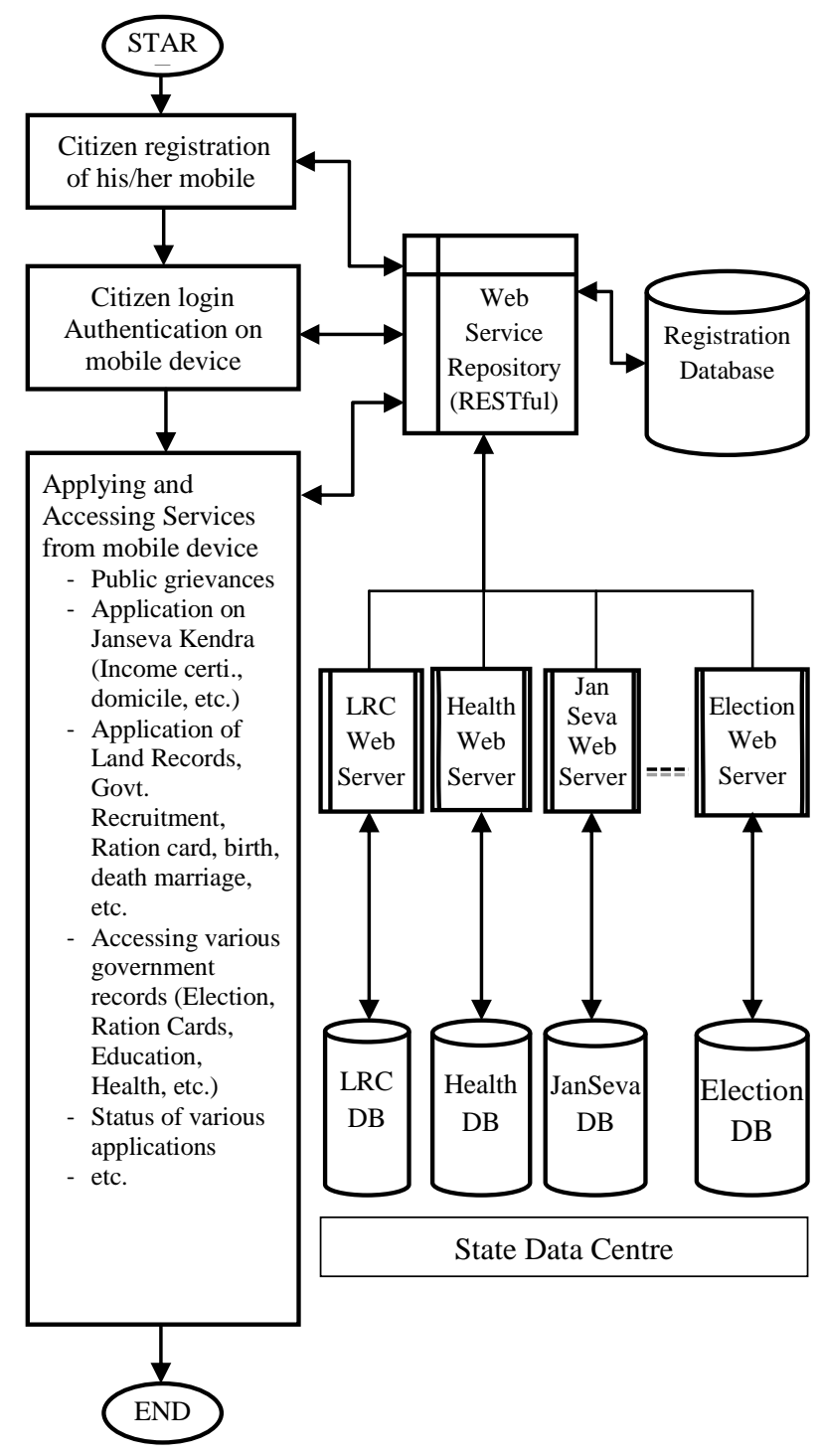

Fig.3. Comunication and Data Flow

Which is most appropriate option from above four options to develop mobile interface will be based on particular requirements. Continuous internet connectivity is required in case of Responsive, Web and Hybrid application, and speed is not satisfactory as provided by native application. From the performance point of view, native application is best and can use local feature of mobile device such as local memory to store data and when gets connectivity it can be upload on remote server. So it provides good performance, citizen centric interface design and cost effectiveness also. Therefore, in this framework native mobile application development is suggested with using network connectivity in backend as and when required to post and get data from remote server.

Web service is used to transfer data between mobile devices and remote server running on different platform. There are different technologies in web service such as SOAP, REST, etc. which one is best for mobile application to communicate with server?. Cesare Pautasso et.al [19] has stated that RESTful HTTP web service is good enough to interact without any extension with process execution engines and their processes and tasks published as resources. It is light weight than SOAP so good to interact with mobile application. RESTful web services [20] are web application based on REST architecture style of application in which data is represented, accessed and modified on the web. In this architecture data and functionality are considered as resources and exposed by web URIs.

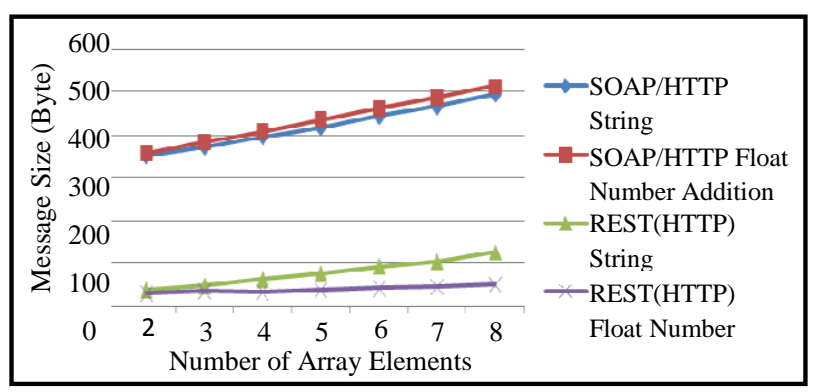

Fig.4. RESTful vs. SOAP Message Sizes of String Concatenation and Float Addition

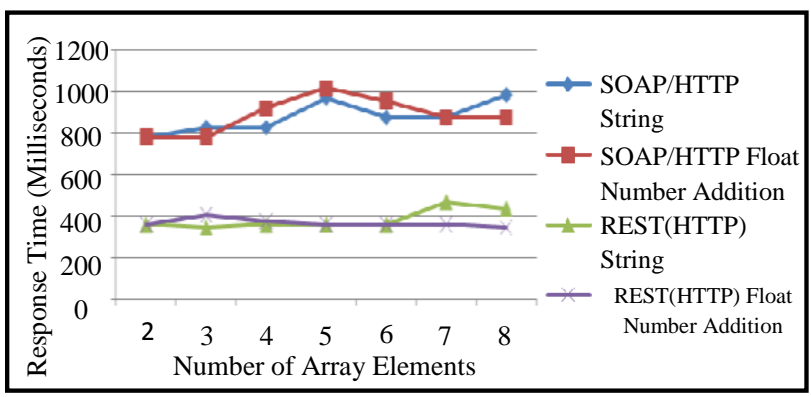

Fig.5. RESTful vs. SOAP Response Time of String Concatenation and Float Addition

An experimental result of Hatem Hamad et.al [21] shows that the message size of RESTful web service is smaller than messages of SOAP web service while communicating. Also the response time in milliseconds of RESTful web service is smaller than messages of SOAP web services. Response time gap is very large between RESTful and conventional SOAP web service. Below figures shows the comparison of message size and response time in the experiment of string concatenation and float addition services using RESTful and SOAP 
based web service.

Due to less Message size and response time RESTful web service requires less processing and transmission time which leads to lower power consumption. This satisfies the physical constraints of mobile devices and achieves the quality of service goal. These results of experiment in [21] support that RESTful web service is recommended for mobile devices. Therefore, REST offers a perfectly good solution for the majority of implementations with greater flexibility and lower overhead. The result of performance comparison between conventional SOAP and RESTful shows that the RESTful provides obviously high performance over SOAP. Therefore, in this integrated mobile governance framework RESTful web service is used for data communication between mobile device and remote server.

\section{B. Implemenntation Platform}

Below platforms are used for the prototype development as a result of the framework.

On client side: Android Operating System, Eclipse development environment for Android, JAVA and SQLite to store local data on mobile device.

The main advantage of adopting Android is that it offers a unified approach to application development. In the world of smartphones, device manufacturers see Android as their best hope to challenge the onslaught of the iPhone, which already commands a large base of applications [22]. Google provides all of the stuff and libraries need to get started building Android applications for free. We can build our Android apps on Macs, Windows, or UNIX and publish apps without anyone's approval [23].

On remote server side: Windows Operating System and IIS web server to host web services, RESTful web service architecture, .NET WCF, C\# and SQL Server to store central database (service records)

Microsoft is strongly committed to REST moving forward [24]. Microsoft WCF 3.5 makes it easy to support either REST or SOAP based on business requirement. Microsoft introduced the new "Web" programming model from .net framework 3.5 by [WebGet], [WebInvoke], UriTemplate, and SyndicationFeed lowers the bar for authoring RESTful services. Also with the introduction of the new WCF REST Starter Kit, they have lowered the bar even further. The project templates make it possible to get RESTful services up and running in minutes [24]. Web API gives all the benefits of the net framework and deals with a lot of the complexities of content negotiation, model binding etc. This Web API can be used to develop RESTful service on Microsoft platform [25].

\section{Designing client side functionality modules}

To develop the client side functionality, several interface modules are designed and programmed in
JAVA to run on Android based mobile devices. This is a native mobile application which includes interfaces for device registration and login, creation of citizen profile, citizen centric service selection for online application, submission of application to government authority and get application status or its progress. All these modules are hyper linked with each other and operated in two languages English and Gujarati as selected in login interface, more languages can be added in the same manner. Below are the operating steps of all these client side functionality modules.

1. Citizen have to register first to get PIN then login by entering mobile number, selecting language and giving PIN.

2. Citizen have to create his profile which is store in local device memory.

3. To apply for service or sumit application, select service category and name of service.

4. Application submittion interface screen displayed with all service related parameters with it's value from citizen profile. User just have to click on "submit" button to submit application on remote server.

5. After submitting service application, user can get status of his application by selecting application number which is stored in mocal device memory at the time of submit application.

Below figures $6,7,8,9$ and 10 show these interfaces to perform all these functionalities in user friendly way.

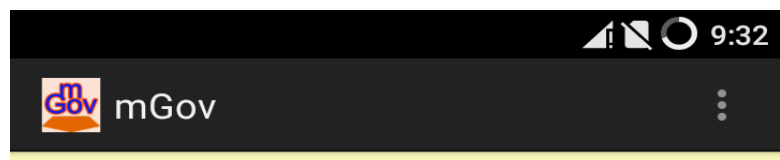

Welcome to mobile governance...

Your device Id :

911436250582727

Your mobile number :

9825765818

Language : English $\bigcirc$

Enter your PIN :
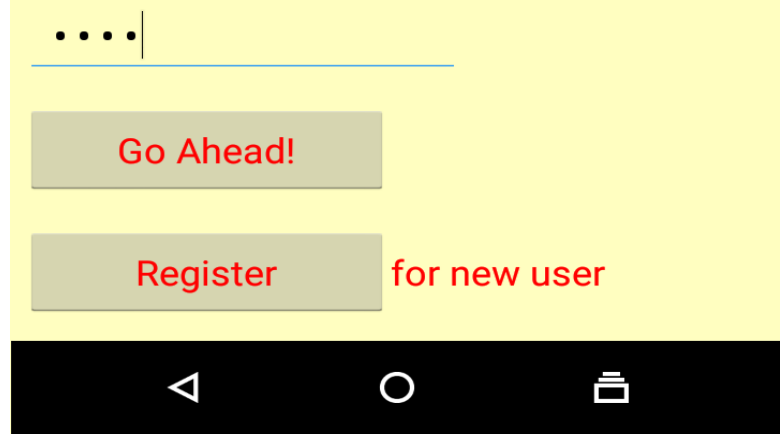

Fig.6. Citizen Login and Authentication Interface 


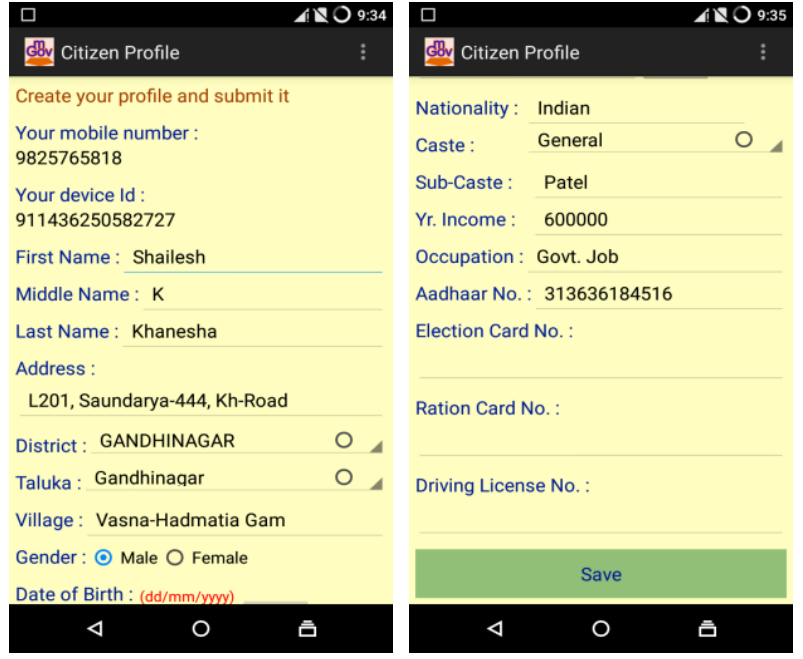

Fig.7. Interface of Citizen Profile Creation

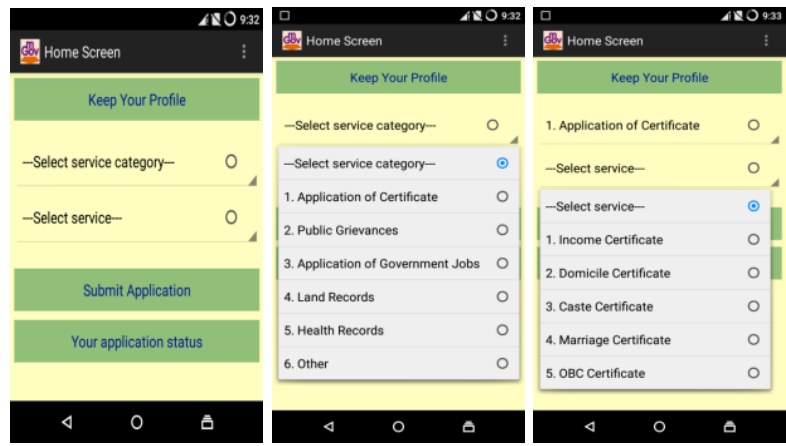

Fig.8. Interfaces of Service Selection to Apply

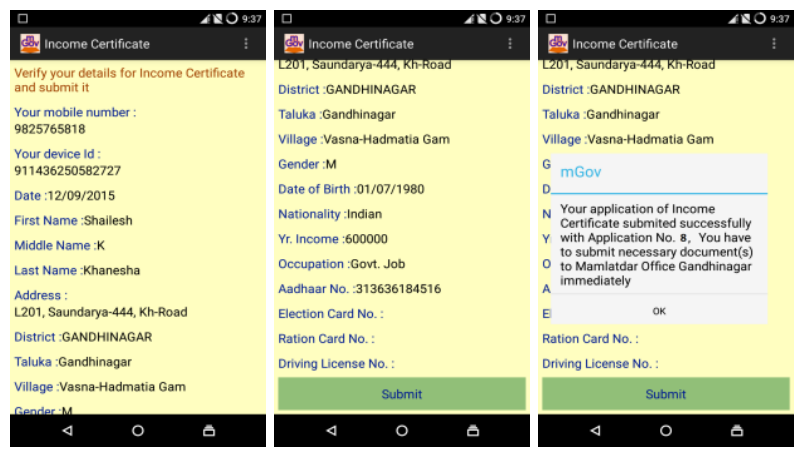

Fig.9. An interface to Apply for Income Certificate

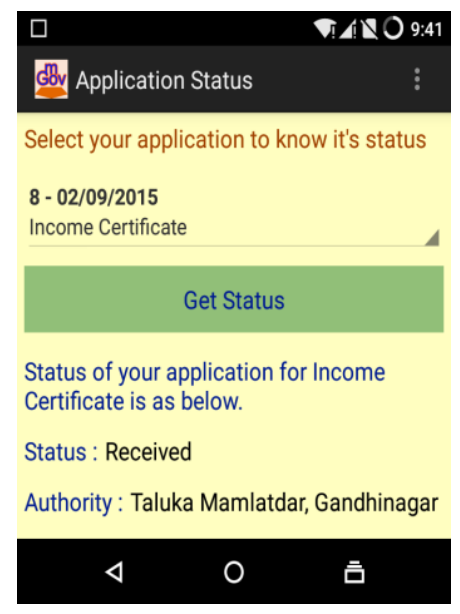

Fig.10. Interface to Know Application Status

\section{Remote server side functionality development}

Communication done between mobile client and server is through RESTful web services API hosted on remote server. In the prototype model, REST architecture is implemented in Microsoft.Net framework. Below screen shot shows the web service methods or functions developed for prototype model of the framework, more methods or functions can be incorporated based on the requirements to create a web service repository specifically for mobile governance framework.

\section{Operations at http://localhost/mGov_Services/Service_Reg.svc

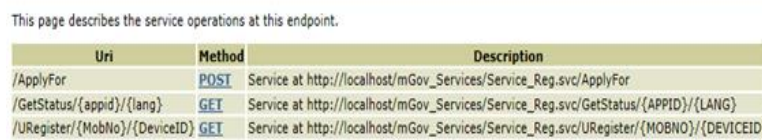

Fig.11. Web Service API Methods

"URegister" method is used to register the mobile device from the client interface. Another important method is "ApplyFor" which is used to send data of citizen application from mobile client using HTTP POST method. Through POST method this web service received the data from client and send Application ID back to the client as response. "GetStatus" method is used to get the status of application on citizen's mobile device.

All the applications for services done by citizens stored centrally in server, its status are updated by various concern e-Governance systems automatically or manually by the government authority. So easy monitoring of all the service delivery is made possible centrally by developing such web based system to generate statistical based MIS reports. Below screen shot shows the inward register entries of applications received from the mobile client, it shows status off each application on single screen.

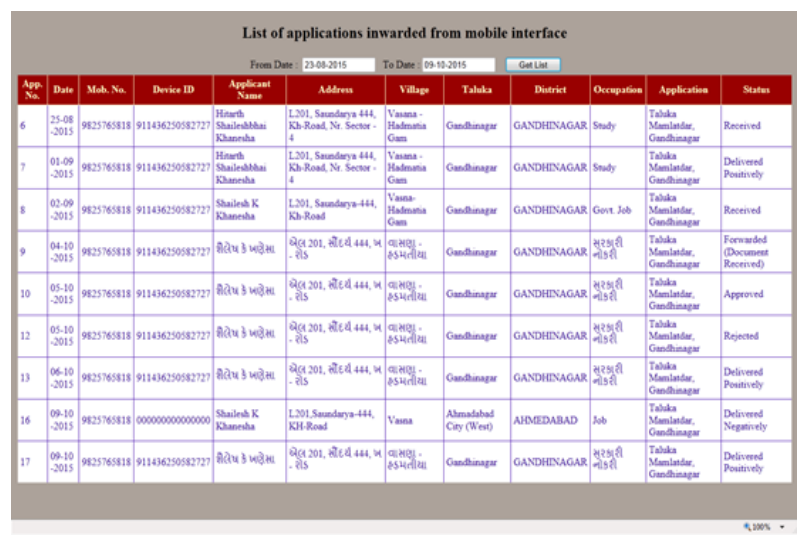

Fig.12. Inward Application List with Its Status

\section{E. Hosting and testing of conceptual framework}

Client side portion of the prototype model is $\mathrm{mGov}$ mobile application which is developed using eclipse for the Android environment. After compiling this application, .apk file of mobile application is created in 
BIN folder of the project. This .apk file is copied into mobile YU5010 and installed for validation. When it installed and run for the first time, master records into mobile database (SQLite) generated automatically such as District, Taluka, Services and other related tables.

Server side portion of the prototype model is RESTful web services which are developed in .NET WCF framework and hosted on IIS (Internet Information Services) in local development machine for testing purpose. Central database behind the web services are hosted on SQL Server on the same machine.

Now, after successfully implementation of client and server side components, there is requirement of wireless connectivity between client and server to validate the prototype model. Therefore, laptop with $\mathrm{Wi}-\mathrm{Fi}$ enabled is taken for hosting of server side components and create a wireless network named "sknet" into it by using virtual router. Here "sknet" is considered as virtual network or internet in which government web services for mobile governance are accessible through mobile device. Virtual router assigned 192.168.173.1 IP address to the host computer in which RESTful web services are hosted for purpose of testing. This IP in referred while developing client module to call the web services. Thus, for successful implementation of conceptual framework, below components are to be ready for testing the framework.

- Mobile device with installed client application and enabled network connectivity.

- Web service hosting setup with central database on server.

- Wireless communication channel (internet or Wi$\mathrm{Fi}$ ) between mobile client and remote server.

\section{RESULT}

In the proposed framework, certain parameters are focused to improve into it. The prototype successfully implemented a subset of the conceptual functions from the functional framework for the mobile governance. The prototype used the functional modules to satisfy the requirements of the use cases. Below table shows observations on these parameters noted while validating the framework in smart phone mobile YU5010 which has 2GB RAM and Quad core 1.2 GHz processor.

Table 1. Result of MAIFCCESS with other reviewed frameworks which has scope of imporvements.

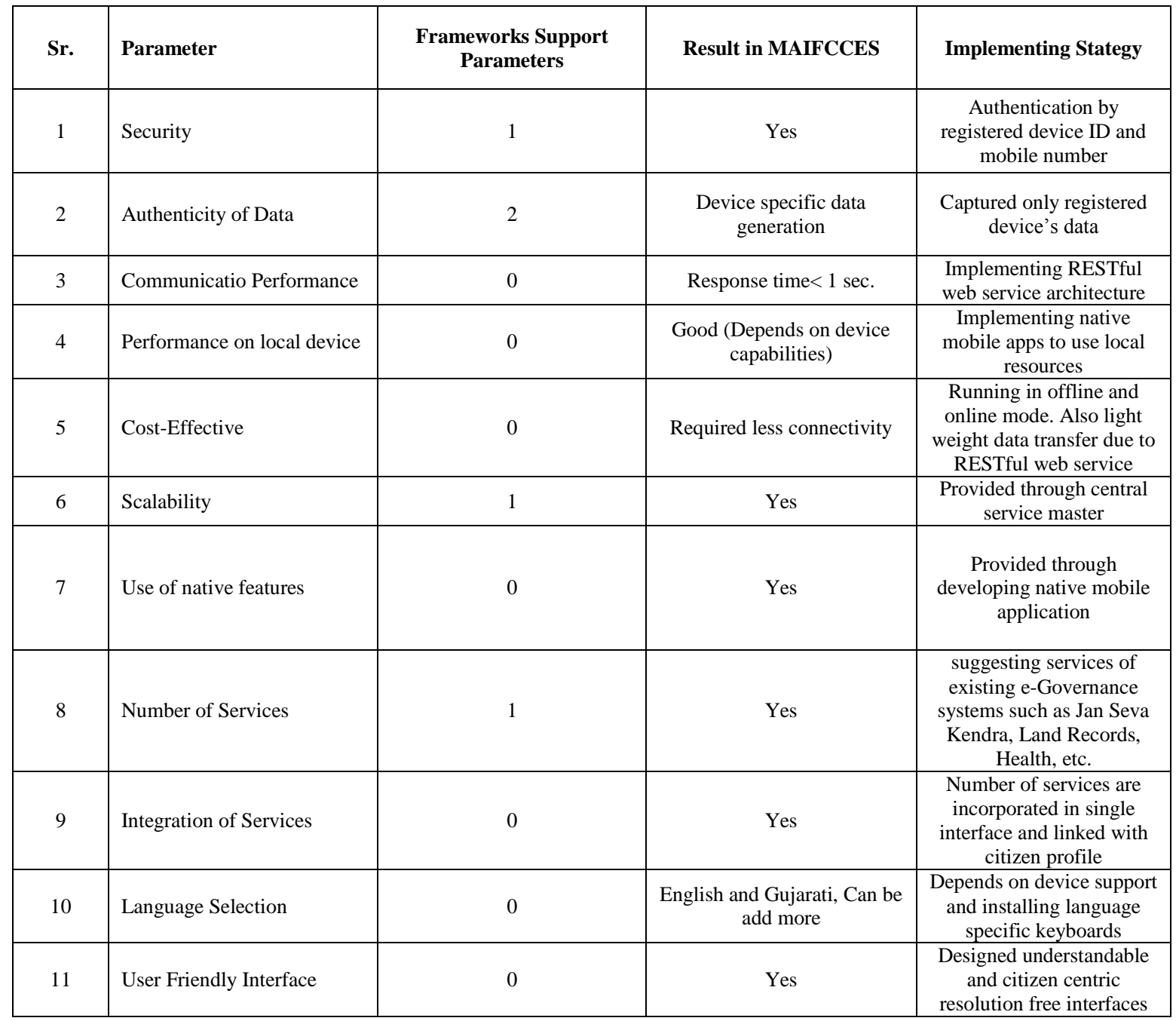


Above table shows that very few mobile governance frameworks (out of seven reviewed frameworks) support the various parameters and others have scope of improvements and not specified some of the parameters. Below fig. 13 shows that MAIFCCESS supports all the parameters which improved overall mobile governance activities.

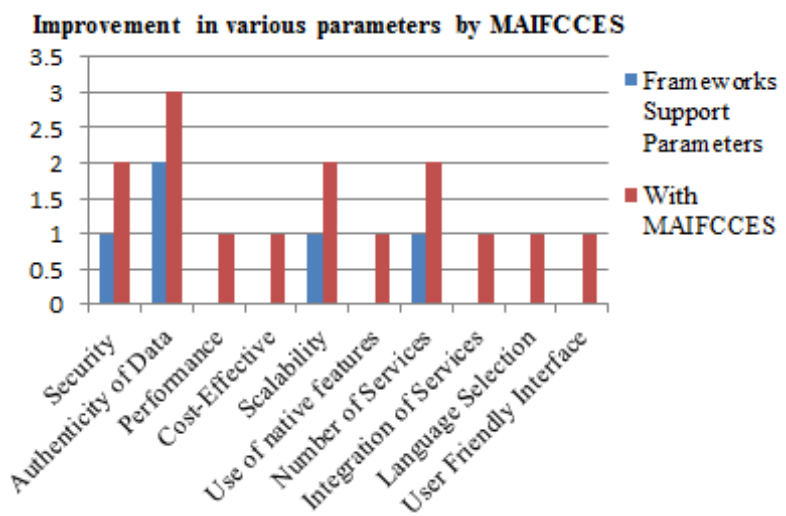

Fig.13. Result with MAIFCCES

\section{CONCLUSION}

The proposed framework providing a government service delivery tool which is useful to citizen for avail various government services and accessing concern records (Land, Health, Education, etc.) from government authority on their mobile device anywhere and anytime. The proposed framework is an integrated service delivery mechanism among citizen and various government departments. Also overcomes problems with existing mGovernance models and provides a foundation for the research, analysis, development and discussion of mobile governance. The prototype provides an example of how the new framework can be used for citizen centric service delivery to alleviate inconsistent communication and confusion regarding $\mathrm{m}$-Governance functionality. This research is limited in that the new framework has only been applied to a single proof-of-concept implementation, which does not include all of the functional components. Additional implementations of the functional modules and their application to more use cases will be useful in further validating the framework and confirming its usefulness in practical implementations.

\section{FUTURE EXTENSION}

Security is the important parameter that can be further improve, such as the use of encryption for the validation and protection of data while communicating with server. Other future work can be focused on integrating this model architecture with Aadhaar database (Citizen's unique identity database with biometric demographic details and maintained by government authority) and Aadhaar based software systems. Digital locker is Aadhaar based software system which kept the scan documents of citizen such as School/Collage certificates, Ration card, Driving license, Election card, passport, etc. and can be accessed by citizen from anywhere and anytime with digitally signed based on Aadhaar database. If Digital locker is integrated with this framework using Aadhaar number then no need to provide physical document by citizen to the government for verification and process the service application.

\section{REFERENCES}

[1] "The Impact of Mobile Government in Organizations: Promises and Pitfalls", Mircea GEORGESCU, The Annals of The "Ştefan cel Mare" University of Suceava. Fascicle of the Faculty of Economics and Public Administration, Vol. 10, No. 2(12),2010, pp.15-22.

[2] "Government Use of Mobile Technology", Barriers, Opportunities, and Gap Analysis, CIO Council, Product of the Digital Services Advisory Group and Federal Chief Information Officers Council, December 2012.

[3] "Effective Development of Mobile Government; Governance Approach", Noor Mohammad Yaghoubi, Mojtaba Pahlavani, Mehrafarin Bakhshimazde, International Journal of Business and Development Studies Vol. 4, No. 1, (2012) pp.87-100.

[4] Sabarish.K and Dr. R. S. Shaji, "A Scalable Cloud Enabled Mobile Governance Framework", IEEE Global Humanitarian Technology Conference, Sep. 26-27, 2014, Trivandrum, pp. 25-34.

[5] Aarathi Prasad, Ronald Peterson, Shrirang Mare, Jacob Sorber, Kolin Paul, and David Kotz, "Provenance framework for mHealth", IEEE, 2013.

[6] Richard K. Lomotey and Ralph Deters, "Mobile-Based Medical Data Accessibility in mHealth", 2nd IEEE International Conference on Mobile Cloud Computing, Services, and Engineering, 2014, pp. 91-100.

[7] Desta Mengistu, Hangjung Zo, Jae Jenug Rho, "MGovernment: Opportunities and Challenges to Deliver Mobile Government Services in Developing Countries", 4th International Conf. on Comp. Sci. and Convergence IT, 2009, IEEE Computer Society, pp.1445-1450.

[8] Shashank Garg,Diatha Krishna Sundar,Isha Garg, "MGovernance:A Mobile Computing Framework for Integrated Disease Surveillance in India", http://grchina.com/mobility/lab/Archives/EuromGov2005 /PDF/21_R357GS.pdf, Dt. 02/06/2015.

[9] Diatha Krishna Sunder, Shashank Garg, "MGovernance:A Framework for Indian Urban Local Bodies",http://www.m4life.org/proceedings/2005/PDF /41_R359SK.pdf, Dt. 04/06/2015.

[10] Emmanuel Eilu, Rehema Baguma and John Sören Pettersson, "M-voting in developing countries: Findings from Uganda", Commonwealth Governance Handbook 2014/15, pp. 25-28.

[11] Department of Electronics and Information Technology, Govt. Of India, The National Mobile Governance Initiative,Mobile Seva, https://www.mgov.gov.in/ msdpbasic.jsp and https://www.mgov.gov.in/index.jsp, Dt. $10 / 06 / 2015$.

[12] Department of Electronics and Information Technology, Govt. Of India Framework for Mobile Governance, Document No: FMG: 01, Version:1.0,January,2012, https://egovstandards.gov.in/sites/default/files/Framework _for_Mobile_Governance.pdf, Dt. 12/06/2015.

[13] Vidya Naik, "Governance goes mobile in Karnataka", March of Karnataka, January 2015, pp. 4-7. 
[14] "Karnataka Mobile One: Pride of India", Posted by: Elets News Network (ENN),eGov Magazine, June 2,2015, http://egov.eletsonline.com/2015/06/karnatakamobileonep ride-of-india/, Dt. 19/06/2015.

[15] "UPOne", https://apps.mgov.gov.in/descp.do? appid=597, 22/06/2015.

[16] Sitwat Langrial, Tuomas Lehto, Harri Oinas-Kukkonen, Marja Harjumaa and Pasi Karppinen, "Native Mobile Applications for Personal Well-Being: A Persuasive Systems Design Evaluation" (2012). PACIS 2012 Proceedings, Paper http://aisel.aisnet.org/pacis2012/93.

[17] Andre Charland and Brian Leroux, "Mobile Application Development: Web vs. Native", Communications of the ACM, May 2011, Vol. 54, No. 5, pp. 49-53.

[18] "Mobile app vs mobile website design: Your four options", Paul Boag,Mon 4 Feb 13, https://boagworld.com /mobile-web/mobile-app-vsmobile-website-design/, Dt. 04/07/2015.

[19] "RESTful Web Services: Principles, Patterns, Emerging Technologies", Cesare Pautasso, Erik Wilde, ECOWS 2010, 8th European Conference on Web Services, Cyprus.

[20] "Architectural Styles and the Design of Network-based Software Architectures", Roy Thomas Fielding, PhD Dissertation, University of California, Irvine, California, USA, 2000.

[21] "Performance Evaluation of RESTful Web Services for Mobile Devices", Hatem Hamad, Motaz Saad, and Ramzi Abed, International Arab Journal of e-Technology, Vol. 1, No. 3, January 2010, pp. 72-78.

[22] Wei-Meng Lee, "Beginning Android 4 Application Development", John Wiley \& Sons, Inc., 2012, pp. 1-9

[23] Jonathan Simon, "Head First Android Development", O'REILY, 2011.

[24] "A Guide to Designing and Building RESTful Web Services with WCF 3.5", Aaron Skonnard, Pluralsight,Oct-2008, https://msdn.microsoft.com/en-us/ library/ dd203052.aspx, dt. 10/08/2015.

[25] "Introduction to REST and net Web API", Martin Kearn, $5^{\text {th }} \quad$ Jan-2015, http://blogs.msdn.com/b/martinkearn /archive/2015/01/05/introduction-to-rest-and-net-webapi.aspx, dt. 10/08/2015.

\section{Authors' Profiles}

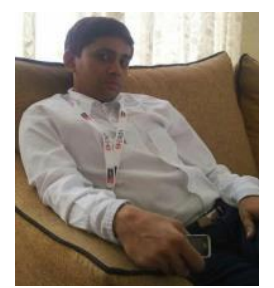

Shailesh K. Khanesha borned at Amreli(Gujarat), India, $1^{\text {st }}$ July 1980. After graduating in computer science (B. Sc.(Computer)) from Saurashtra University, Rajkot(Gujarat), India in 2000 he took his master degree in science with majors in IT and Computer Application (M. Sc. IT\&CA) from Saurashtra University, Rajkot(Gujarat), India in 2002. Recently he is doing M. Phil. in the area of "Mobile Governance" from KSV University, Gandhinagar(Gujarat), India since 2015.

$\mathrm{He}$ is Scientist-B and System Analyst in National Informatics Centre(NIC), Government of India, Gandhinagar(Gujarat), India. He joined NIC in Jun-2006 and engaged in many state and national level e-governance projects. He was lecturer for computer science subjects in Smt. K. P. Dholakiya Infotech Mahila College, Amreli(Gujarat), India from Jun-2002 to Jun2006. He has attend many national conference and submitted articles on e-Governance, Cryptography and Secure Communication, Robotics and Wireless Communication. His current area of research are e-governance, m-governance, mobile computing and government process re-engineering.

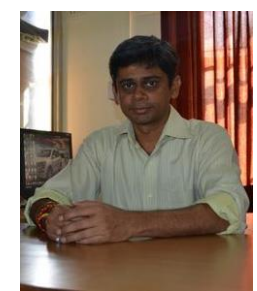

Dr. Ashish N. Jani borned at Morbi(Gujarat), India, $6^{\text {th }}$ Nov 1976. After graduating in physics (B. Sc.(Physics)) from Saurashtra University, Rajkot(Gujarat), India in 1999 he took his master degree in science with majors in information technology (M. Sc. IT) from Punjab Technical University, India in 2003 and completed Ph. D. computer science from Hemchandracharya North Gujarat University, Patan(Gujarat), India in 2007. Also he has done Post Doctoral Research - FAU USA.

$\mathrm{He}$ is Assistant Professor in S K Patel Institute of Management \& Computer Science (MCA), KSV University, Gandhinagar(Gujarat). His current area of research are .Net, Embedded System, Computer Vision, Web Development and Mobile Computing.

How to cite this paper: Shailesh Khanesha, Ashish Jani,"Mobile Augmented Integrated Framework for Citizen Centric E-Governance Services-MAIFCCES", International Journal of Information Engineering and Electronic Business(IJIEEB), Vol.8, No.3, pp.47-56, 2016. DOI: 10.5815/ijieeb.2016.03.06 